Mémoire.

\title{
PREMIER CAS DE TOXOCAROSE HUMAINE (?) A LOCALISATION CIRCULATOIRE DE VERS ADULTES, DÉPISTÉE PAR LA PRÉSENCE D'CEUSS EMBRYONNÉS A L'HÉMOLYSE CONCENTRATION
}

\author{
J. LAPIERRE*, J. PETITHORY**, C. TOURTE-SCHAEFER*, TRAN VINH HIEN*, \\ C. HOLLER *, E. LEBAS SAISON*, J. RONDIER ***, P. BOUCHACOURT ***
}

\begin{abstract}
RÉSUMÉ
A l'occasion d'une recherche de microfilaires Loa loa par hémolyse concentration sanguine chez un Camerounais présentant une hyperéosinophilie, des œufs d'ascaridés évoquant la morphologie de Toxocara canis ou $T$. cati sont découverts et retrouvés en nombre variable lors de six recherches successives dans les sept semaines suivantes. Ces œufs mesurant de 100 à $112 \mu \times 75$ à $100 \mu$ sont embryonnés à divers stades allant pour certains jusqu'à l'éclosion observée sous le microscope, libérant des larves de 437 à $504 \mu$. Ces phénomènes font supposer l'existence de vers adultes
\end{abstract}

dans un biotope riche en oxygène du système circulatoire (ventricule gauche?). L'existence d'un incident pulmonaire (épauchement pleural) est difficile à mettre au compte certain de la parasitose (3\% de polynucléaires éosinophiles seulement dans le liquide de l'épanchement). Des traitements par thiabendazole (pour cure d'une anguillose associée) et diéthylcarbamazine sont bien tolérés, le premier s'avérant limité voire nul sur «l'ascaridiose sanguine " alors que le second entraîne la disparition définitive des œufs dans les prélèvements de sang effectués à six reprises jusqu'au sixième mois.

Summary: First case of human toxocarosis (?) with localization of adults in the circulatory system, diagnosed by the presence of embryonated eggs in blood concentration.

Ascarids's eggs were found by blood concentration in a Cameroonese patient with hypereosinophilia. Eggs, similar to those of Toxocara canis or cati, were present in 6 blood concentrations carried out during 7 weeks. These eggs $(70-75 \times 100-112 \mu \mathrm{m})$ contained embryos and hatching of 437-504 $\mu \mathrm{m}$ long larvae was sometimes observed. The presence of adult worms in a well oxygenated localization (e.g.; the left ventricle) was supposed. A pleural effusion was also observed but the low number of eosinophils in the pleural fluid ( $3 \%$ ) eliminated the responsability of the parasite. The treatment by thiabendazole for an associated strongyloidosis was well tolerated but inefficient. On the contrary, the treatment by diethylcarbamazine, well tolerated, induced the disparition of the circulating eggs. This result was confirmed by 6 blood concentrations carried out during 6 months.
Individualisé par Beaver $(1,2)$ chez l'enfant en 1952, le syndrome de "Larva migrans viscérale » (toxocarose) est dû à la migration prolongée dans l'organisme humain de larves infectantes de Toxocara canis voire de Toxocara cati. Souvent bien tolérée, la présence de ces larves peut néanmoins déterminer de graves complications cérébrales, oculaires ou cardiaques $(5,20)$.

En fait; les larves d'ascarides animales pouvant déterminer chez l'homme des cas de « Larva migrans viscérale » sont nombreuses $(9,10,15,19,22)$. Elles peuvent provenir d'animaux domestiques (chat, porc, équidés, bovidés),

* Service de Parasitologie et Médecine tropicale (Pr LAPIERRE), Hôpital Cochin, 27, rue du Faubourg-Saint-Jacques, F-75674 Paris Cedex 14.

** Service de Biologie ( $\mathrm{D}^{\mathrm{r}}$ Petithory), CHR de Gonesse, F 95500 Gonesse.

*** Service de Rhumatologie (Pr Delbarre) $\dagger$, Hôpital Cochin, Paris.

Accepté le : 29 mars 1991. d'animaux sauvages (mustélidés, divers) (11), voire peutêtre de serpents (23). Mais de toutes les espèces évoquées, l'ascaris du chien semble être cependant le plus souvent en cause. De nombreux cas ont été signalés dans le monde, surtout chez l'enfant $(1,12)$, mais également chez l'adulte $(15,30)$.

Par contre, le parasitisme de l'homme par des ascaridés adultes d'animaux reste rare car les ascaridés font preuve à l'état adulte d'une spécificité étroite vis-à-vis de leur hôte. Les cas signalés dans la littérature de parasitisme intestinal humain par $T$. canis et $T$. cati, voire d'autres espèces sont rares $(3,7,8,17,18,29,16)$. Certains auteurs (16) s'étonnent cependant du peu de cas décrits chez l'homme et émettent l'hypothèse que des ascaris du chien ou du chat ont été considérés comme des formes immatures d' $A$. lumbricoides.

En tout cas, à notre connaissance, aucune localisation de vers adultes du genre Toxocara dans le système circulatoire humain n'a été décrit ainsi que cela semble être le cas dans l'observation que nous relatons. 


\section{OBSERVATION}

M. L. Manfred, 26 ans, musicien camerounais, en France depuis 1966, mais ayant effectué depuis des déplacements professionnels au Zaïre 1968, au Tchad 1969, Cameroun en 1970, États-Unis en 1973, est hospitalisé dans le service de rhumatologie de l'hôpital Cochin ( $\mathrm{P}^{\mathrm{r}}$ Delbarre), le 2 janvier 1974, pour spondylodiscite dorsale.

Chez ce malade sans antécédents notables, le début des troubles remonte au 15 avril 1973 par l'apparition, quinze jours après un traumatisme, de douleurs dorso-lombaires en ceinture; les radiographies rachidiennes et la NFS sont normales, la VS à $7 \mathrm{~mm}$ à la $1^{\text {re }}$ heure. Fin juillet 1973 , le tableau se modifie en raison de l'apparition d'une fièvre à $38^{\circ}$ et de l'aggravation des douleurs rachidiennes qui réagissent de plus en plus mal aux traitements antalgiques banals. Un nouveau bilan pratiqué en novembre 1973 ayant montré une VS à $40 \mathrm{~mm}$ à la $1^{\text {re }}$ heure, le malade est hospitalisé.

A son admission, le diagnostic de spondylodiscite très vraisemblable cliniquement, sera confirmé par les radiographies du rachis qui montrent une double atteinte D8-D9-D10 associée à une bascule de $\mathrm{D} 9$, à une spondylite de $\mathrm{D} 7$ et une image d'abcès paravertébral.

Les examens complémentaires apportent les éléments suivants : NFS : GR : 4060000, GB : 10900 dont PN : 81, PE : 6, L : $12, \mathrm{M}: 1$, plaquettes : 180000 , VS : $60-96$, glycémie : $0,85 \mathrm{~g} / \mathrm{l}$, urée sanguine : $0,35 \mathrm{~g} / \mathrm{l}$, cutiréaction +++ , recherches de BK dans les crachats et les urines négatives, radiographie pulmonaire normale. La recherche de parasites dans les selles est alors négative (voir ci-après).

L'étiologie tuberculeuse sera affirmée par la biopsie chirurgicale du foyer vertébral montrant histologiquement des follicules tuberculeux typiques et permettant l'évacuation d'un pus qui s'avèrera riche en $\mathrm{BK}$ sur les cultures.

Le lendemain de l'intervention (14 janvier 1974) est débuté un traitement associant $600 \mathrm{mg}$ de Rifamycine ${ }^{\circledR}, 600 \mathrm{mg}$ d'isoniazide (Rimifon ${ }^{\circledR}$ ) et $1200 \mathrm{mg}$ d'éthambutol; ce traitement sera bien toléré sur le plan général hormis une ascension modérée et transitoire des phosphatases alcalines et des transaminases, et s'accompagnera d'une amélioration clinique et biologique rapide et persistante.

Le 11 février, l'apparition d'une douleur thoracique droite fait découvrir un épanchement pleural confirmé par la radiographie; la ponction ramine $500 \mathrm{~cm}^{3}$ d'un liquide séro-sanglant et une nouvelle radiographie pratiquée le 13 février met en évidence une opacité segmentaire du lobe inférieur droit, dont le caractère systématisé est très évocateur d'une atélectasie pulmonaire. L'examen du liquide pleural montre des protides à $59 \mathrm{~g} / 1$, un sucre à $1,15 \mathrm{~g} / 1$ et $15000 \mathrm{GR} / \mathrm{mm}^{3}, 2500$ leucocytes $/ \mathrm{mm}^{3}$ dont $30 \%$ de PN, $3 \%$ de polynucléaires éosinophiles, $60 \%$ de lymphocytes et $7 \%$ de cellules mésothéliales $/ \mathrm{mm}^{3}$; les cultures sur milieux usuels et sur Lowenstein restent négatives. L'évolution est favorable puisque l'atélectasie qui a disparu dès le 22 février ne sera retrouvée à aucun examen ultérieur; un épanchement pleural modéré persiste jusqu'au 10 mars environ pour disparaître également complètement.

L'hyperéosinophilie sanguine qui s'est élevée à $20 \%$ le 20 février fait évoquer la possibilité de rattacher cet épisode à une origine parasitaire et entreprendre un bilan parasitaire plus étendu.

Un examen sanguin (hémolyse concentration) pour rechercher des microfilaires de Loa loa en raison de l'origine géographique du malade, amène le 21 février 1974 à l'étonnante découverte dans le culot de centrifugation d'une centaine d'œufs d'ascaridés embryonnés à divers stades mesurant 100 à $106 \mu \times 75$ à $87 \mu$ présentant les caractères morphologiques d'œufs de Toxocara canis ou cati. Ces mêmes œufs seront retrouvés en quantité variable lors de 6 recherches successives effectuées dans les 7 semaines qui suivent aussi bien dans le sang veineux qu'artériel (sang fémoral) et la nuit que le jour. A plusieurs reprises, nous avons pu voir éclore ces œufs lors de l'examen microscopique et libérer des larves mesurant environ $500 \mu$ (437 à $504 \mu$ ) (tableau I).

La recherche d'œufs d'ascaris dans les selles à diverses reprises restera toujours négative mais permettra de découvrir successivement des larves rhabditoïdes de Strongyloides stercoralis (anguillules) le 25 février 1974 et quelques œufs de Schistosoma intercalatum (agent de la bilharziose rectale) le 11 mars 1974. La recherche d'œufs d'ascaridés dans le liquide pleural et le liquide d'aspiration bronchique (28 février 1974) sera infractueuse.

L'enquête séro-immunologique parasitaire (21 février) donnera les résultats suivants : réaction d'immunofluorescence indirecte de la filariose (avec utilisation de Dipetalonema vitae comme antigène) légèrement positive au $1 / 20$, de l'amibiase $\pm 1 / 100$ (non significatif), de la toxocarose et de la bilharziose négatives; les réactions d'immunodiffusion (Ouchterlony) avec les antigènes de Fasciola hepatica (grande douve), liquide hydatique, Aspergillus et différents antigènes ascaridiens: Ascaris suum (porc), Parascaris equorum (cheval), Toxocara canis (chien), Ascaris lumbricoides (homme) sont toutes négatives.

Répétées à plusieurs reprises après traitement par le thiabendazole et la Notézine ${ }^{\circledR}$, les réactions séro-immunologiques par immunofluorescence de la toxocarose et par immunodiffusion avec les divers antigènes ascaridiens resteront toujours négatives.

Le bilan immunologique s'avère pourtant normal. Le dosage des immunoglobulines plasmatiques par la méthode de Mancini est normal : IgG $22 \mathrm{~g} / 1(\mathrm{~N}: 14 \mathrm{~g} / \mathrm{l}) ; \operatorname{IgA} 5,7 \mathrm{~g} / 1(\mathrm{~N}: 2,55 \mathrm{~g} / \mathrm{l})$; IgM $1,45 \mathrm{~g} / 1(\mathrm{~N}: 1,65 \mathrm{~g} / \mathrm{l}) ; \mathrm{IgE}: 1480 \mathrm{UI} / \mathrm{ml}$; test à la candidine + au $1 / 1000$; test à la tuberculine +++ à $10 \mathrm{U}$; test de transformation lymphoblastique : témoin $1 \%$; PHA : $93 \%$; tuberculine : 29,6\% (réponse positive).

En présence de cette double parasitose à nématodes (bilharziose rectale exclue), on décide après maintes hésitations de traiter ce malade d'abord de son anguillulose puis de son " ascaridiose" pour essayer de définir ce qui revient à chaque parasitose. Le 21 mars, M. L. reçoit du thiabendazole $(6 \mathrm{cp}$ à 0,500$)$, ce qui fera disparaître les larves d'anguillules dans les selles (coprologie suivie jusqu'au $12^{\mathrm{e}}$ mois) sans toutefois influencer de façon durable l'éosinophilie qui continuera à évoluer en dents de scie (tableau II).

Par contre, le traitement par le thiabendazole sera sans effet sur " l'ascaridiose sanguine " puisque des œufs seront retrouvés lors des hémolyses concentrations effectuées le 4 avril ( 2 œufs atypiques ne mesurant que $40 \mu$ ), le 16 ( 1 œuf non segmenté de $100 \times 87 \mu$ ) et le 19 avril ( 3 œufs ovalaires de $100 \times 90 \mu$ ).

Le 16 avril un traitement par la diéthylcarbamazine (Notézine ${ }^{\circledR}$ ) est entrepris avec posologie prudemment croissante pendant 1 semaine puis de 4 comprimés à $0,10 \mathrm{~g}$ sous couvert de $30 \mathrm{mg}$ de prednisone et de $150 \mathrm{mg}$ de prométhazine par jour pendant 10 jours. En dehors d'une petite éruption et d'un léger prurit, au $2^{\mathrm{e}}$ jour aucun incident ne sera noté. Ce traitement aura pour effet de faire disparaître les œufs d'ascaris du sang circulant dès le 22 avril. Ils ne seront plus retrouvés lors de contrôles ultérieurs effectués à 4 reprises les $22,25,29$ avril et le 5 mai, ainsi que plus tardivement le 28 mai et le 11 octobre (voir ci-après).

Le 6 mai, M. L. part en convalescence au centre héliomarin de Vallauris où il séjournera jusqu'au 13 mars 1975. Grâce à l'amabilité du Docteur Kalachnikoff, le taux de l'éosinophilie sera régulièrement suivi pendant toute la durée du séjour à Vallauris et révèlera une réascension du taux de l'éosinophilie en dents de scie (7 à $27 \%$ ).

A deux reprises, le 28 mai et le 11 octobre, donc un et cinq mois après la cure de Notézine ${ }^{\circledR}$, du sang sera envoyé pour une recherche d'œufs d'ascaris qui s'avèrera négative. Deux examens séro-immunologiques ( 27 mai et 26 septembre) de la toxocarose par immunofluorescence et par immunodiffusion (ouchterlony) avec les antigènes ascaridiens de l'homme, du porc, du chien et du 
TABLEAU I. - Tableau récapitulatif des recherches d'œufs

dans le sang (hémolyse concentration) et l'épanchement pleural.

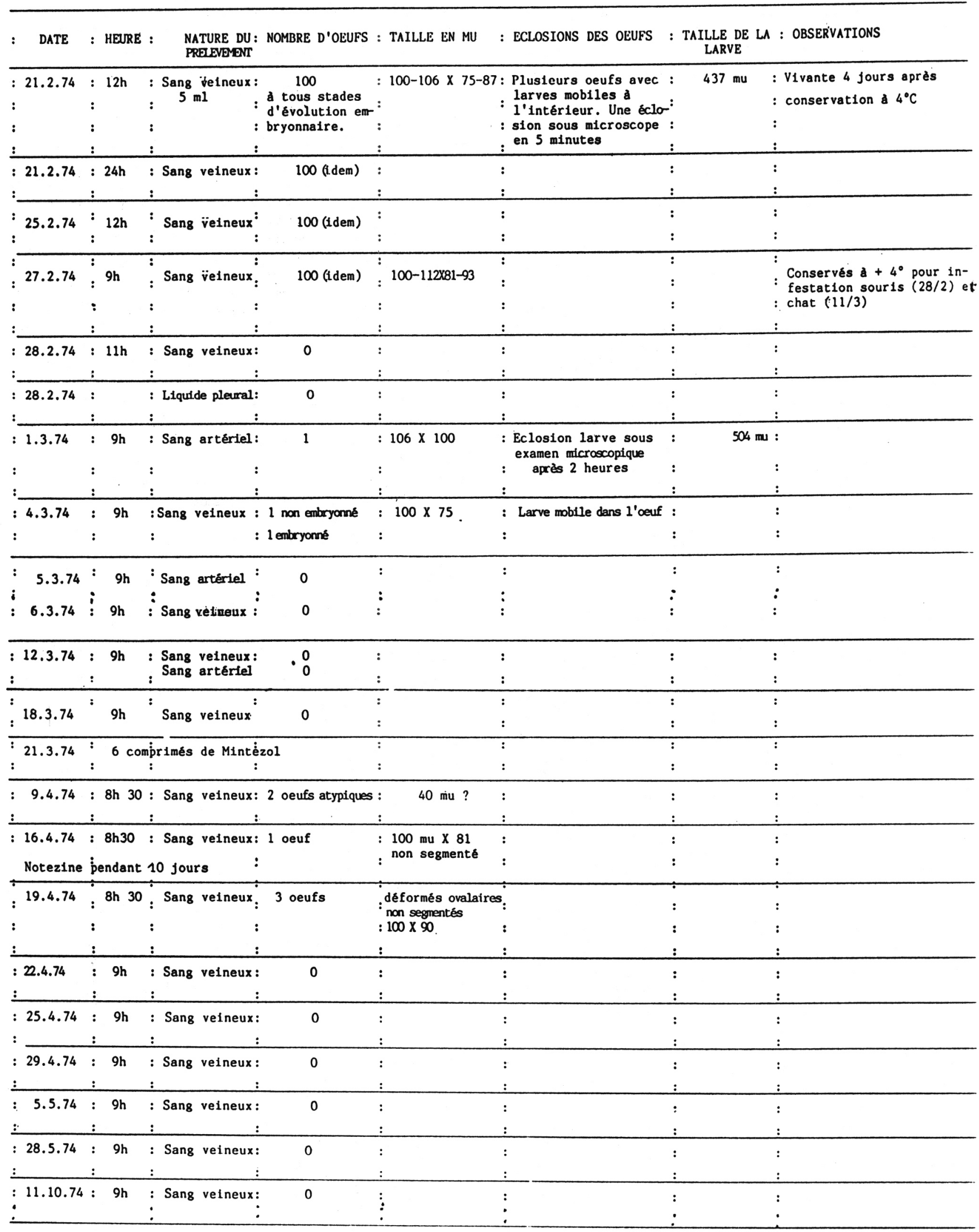


TABlEAU II. - Courbe évolutive de l'éosinophilie, avec correspondance des examens et des traitements effectués.

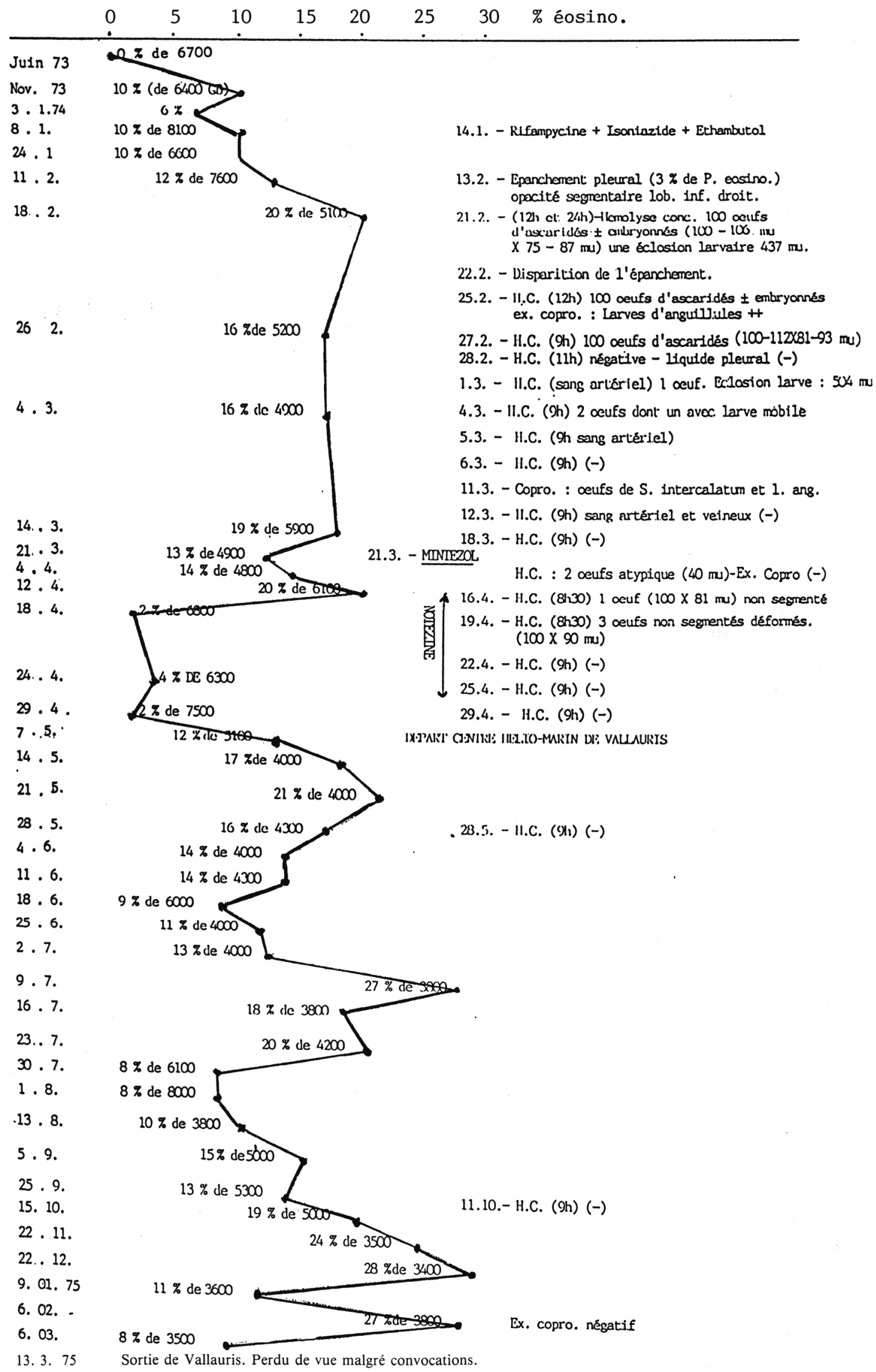




\section{J. LAPIERRE ET COLL.}

cheval seront toujours négatifs. Un examen parasitologique des selles avec extraction de Baermann pour recherche spécifique des anguillules, effectué à Vallauris le 6 février 1975 sera également négatif.

Après sa sortie du centre héliomarin (13 mars 1975), malgré plusieurs lettres adressées, nous n'avons à notre grand regret jamais pu revoir $\mathrm{M}$. L.

Par une lettre du 5 février 1981 du dispensaire d'Hygiène sociale de Saint-Germain-en-Laye nous demandant des renseignements sur des antécédents éventuels de pian à propos d'une sérologie de la tréponématose positive, et son refus de se rendre à leur convocation, nous avons seulement pu savoir que M. L. était toujours en France et en vie à cette date.

\section{DISCUSSION}

Cette observation soulève divers points de discussion.

\section{1 - Le parasite en cause}

D'après l'aspect des œufs (fig. 1, 2, 3), il semble qu'il s'agisse d'œufs de Toxocara (probablement $T$. canis) encore que les dimensions extrêmes (100 à $112 \mu \times 75$ à $100 \mu$ ) des œufs observés soient supérieures aux mensurations moyennes classiques : 75 à $85 \mu$ pour $T$. canis, 65 à $75 \mu$ pour $T$. cati, 75 à $85 \mu$ pour $T$. leonina.

Une tentative d'infecter des chats et des souris par des œufs embryonnés après 24 heures de conservation à $+4^{\circ} \mathrm{C}$ s'est soldée par un échec : pas d'observation de vers adultes chez le chat ni de larves ou de lésions histopathologiques chez la souris lors de l'examen après sacri- fice des animaux. Chez un chat sacrifié au $6^{\mathrm{e}}$ mois, la découverte au niveau du foie d'une plaque scléreuse de $3 \mathrm{~mm}$ de diamètre avait retenu notre attention mais elle ne révélait rien de particulier en coupe histologique. Nous n'avions pas à ce moment de jeunes chiots pour tenter de les infecter.

\section{2 - LOCALISATION DES VERS}

Chez le chien selon l'âge, on peut observer soit un cycle entéro-hépato-pneumo-trachéo-entéral chez le jeune chiot ou un cycle de type entéro-pneumo-somatique chez les chiens de plus de 3 mois. Dans ce dernier cas, les larves sont disséminées en divers tissus. Chez la chienne en gestation, les larves enkystées dans les divers tissus de l'animal peuvent se libérer de leur localisation somatique, migrer dans la circulation placentaire et de là dans l'organisme du fœtus. Après la naissance du chiot, l'évolution larvaire reprendra son cours et les parasites arriveront dans le tractus gastro-intestinal après migration pneumo-trachéale.

Chez les hôtes anormaux, seule la migration somatique pure est observée $(9,10,27)$.

Il est donc probable, après ingestion des œufs par notre malade, que les larves écloses dans l'intestin aient migré par le foie, le cœur droit, l'artère et la veine pulmonaire et soient parvenues dans le ventricule gauche pour y donner des adultes bénéficiant d'une installation confortable et d'une bonne oxygénation.

On peut conclure à l'existence de plusieurs vers adultes
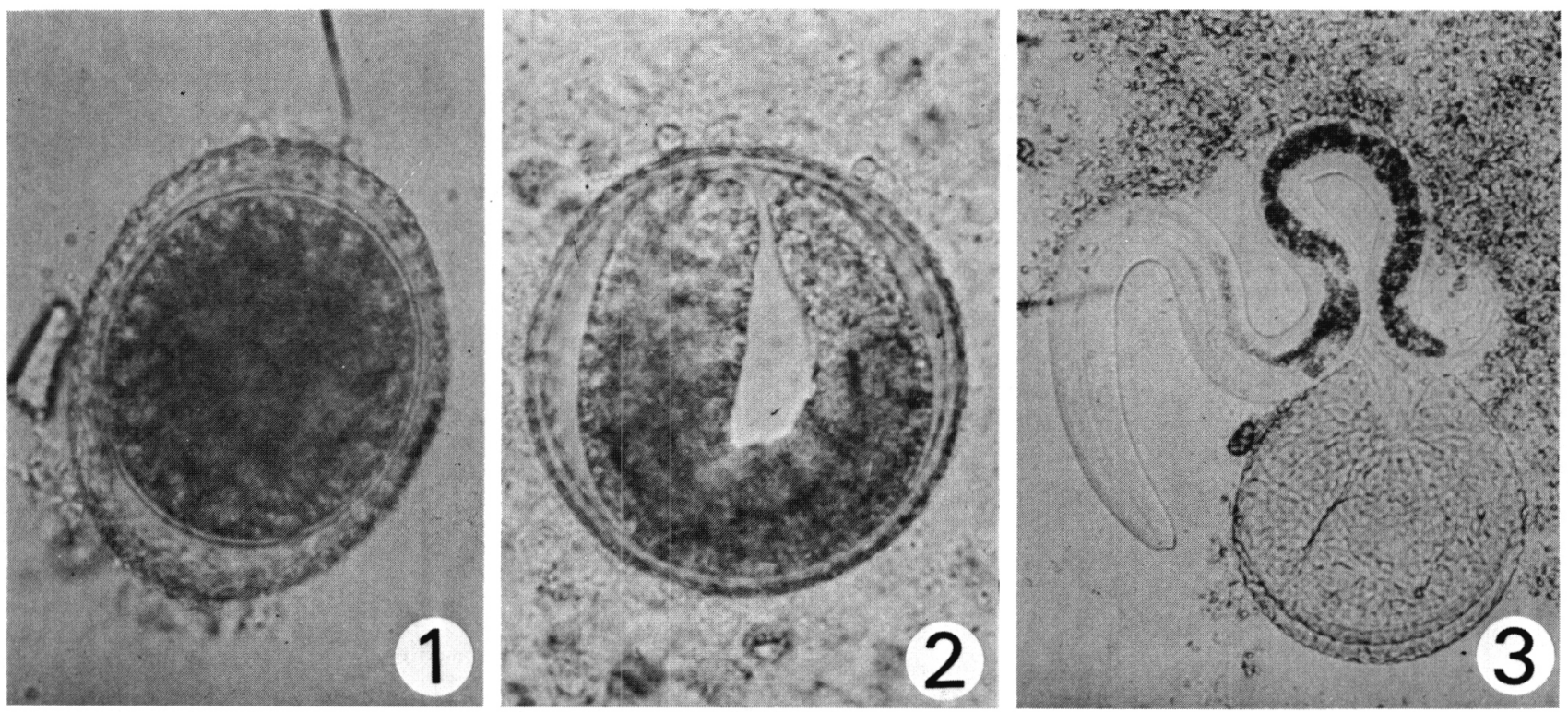

Fig. 1. - Hémolyse concentration du 21 février 1974 : œufs de Toxocara sp.

Fig. 2. - Hémolyse concentration du 27 février 1974 : œufs de Toxocara sp. $(102 \times 100 \mu)$ avec larve mobile interne.

Fig. 3. - Hémolyse concentration du 21 février 1974 : à $12 \mathrm{~h}$, œufs de Toxocara sp. $(100 \times 87 \mu)$ avec éclosion larvaire $(437 \mu)$, cinq minutes après observation microscopique. 
de Toxocara (au moins un mâle et une femelle) puisque les œufs étaient embryonnés.

En ce qui concerne la découverte des œufs dans le système veineux et artériel, elle s'explique par l'existence de nombreuses anastomoses artériosoveineuses pouvant laisser passer des billes de 2 à $300 \mu$ et donc des œufs d'un diamètre sensiblement équivalent à $100 \mu$.

La présence d'œufs embryonnés représente aussi un phénomène inhabituel puisqu'il est admis que les œufs d'ascaridés ne s'embryonnent que dans le milieu extérieur en présence d'oxygène (10). Phan Trinh et coll. $(6,24)$ au Viêt-nam ont cependant signalé l'éclosion larvaire intracorporelle d'œufs d'Ascaris lumbricoides (donc d'ascaris humains) erratiques sur le parcours hépato-pulmonaire. Des œufs aux différents stades d'embryonnement ont été découverts dans le foie, le poumon et le cœur d'un enfant mort de graves abcès ascaridiens. Les auteurs pensent que la communication des abcès hépatiques avec le poumon droit par fistulisation du diaphragme a permis le libre passage de l'oxygène respiré du poumon au foie.

Bien qu'il ne s'agisse pas d'ascaridés animaux mais d'Ascaris lumbricoides, il est intéressant de citer les quelques cas exceptionnels signalés dans la littérature de localisation dans le système cardiovasculaire $(4,6,13,26)$ et notamment celui rapporté par Phuoc et Schmauss (25) dans le service du Professeur Ton That Tung à Hanoï. « Chez une femme de 42 ans, déclaration brusque d'une douleur, précordiale lancinante fugace, suivie immédiatement d'une douleur dans l'extrémité inférieure de la jambe gauche avec apparition d'un œdème bleu noirâtre et absence de pulsation au niveau de l'artère fémorale. La gangrène nécessite une amputation de la jambe au tiers inférieur de la cuisse. Deux ascaris de $30 \mathrm{~cm}$ sont trouvés dans la lumière de l'artère poplitée tandis que dans l'inflammation granuleuse des parois artérielles et des tissus périvasculaires sont découverts des œufs d'ascaris. " Les auteurs évoquent, pour expliquer cette rare complication, une éventuelle persistance du foramen ovale ayant permis le passage du ver du côté droit au côté gauche du cœur puis l'embolisation dans l'artère fémorale.

Plus ancienne (1929) et toute aussi intéressante est l'observation de Boettiger et Werne (4) qui lors d'une autopsie chez un sujet adulte mort d'une thrombose pulmonaire et d'une pneumonie interstitielle chronique ont découvert 2 vers adultes de $10 \mathrm{~cm} \times 5 \mathrm{~mm}$ dans le ventricule droit.

\section{3 - ToléRANCE DU PARASITISMe}

A l'opposé de ces cas associés à des graves complications, on peut opposer la bonne tolérance du parasitisme dans notre observation.

L'accident pulmonaire droit (atélectasie pulmonaire à la radio et épanchement pleural) est aussi difficile à rattacher à une étiologie tuberculeuse (survenue tardive, un mois après le début du traitement associant rifampycine, isoniazide, éthambutol alors que l'évolution était favorable), que parasitaire ( $3 \%$ de polynucléaires éosinophiles seulement dans le liquide d'épanchement pleural). Quoi qu'il en soit, l'évolution de cet accident pulmonaire fut rapidement favorable puisque l'atélectasie avait disparu en une dizaine de jours et l'épanchement pleural définitivement le $20^{\mathrm{e}}$ jour.

A noter aussi malgré nos craintes et peut-être du fait de notre prudence thérapeutique que les traitements par le thiabendazole $\left(\right.$ Mintezol $^{\circledR}$ ) et la diéthylcarbamazine (Notézine ${ }^{\circledR}$ ) ont été très bien supportés.

\section{4 - RÉPONSES IMMUNOLOGIQUES}

A l'exception du test d'immunofluorescence indirecte de la filariose (avec l'antigène Dipetalonema vitae) discrètement mais non significativement positif, toutes les autres réactions séro-immunologiques effectuées par la technique d'Ouchterlony et d'immuno-électrophorèse avec divers antigènes (ovulaires, larvaires, adultes, excrétés-sécrétés) d'ascaridés : Toxocara canis (chien), Toxocara cati (chat), Ascaris vitulorum (veau), Toxascaris leonina (chien), Parascaris equorum (cheval), Ascaris suum (porc); voire d'autres nématodes : Trichinella spiralis, Nippostrongylus, Hemonchus contortus ou d'autres helminthes Fasciola hepatica (Grande douve), Schistosoma mansoni, Poracaecum ensicaudatum (Tenia du merle) ont toutes été négatives aussi bien avant qu'après traitement.

A noter que le bilan immunologique de notre patient (voir dans l'observation) était normal, on s'explique donc mal cette absence quasi totale de réponse immunologique.

\section{5 - ÉVOLUTION DE L’ÉOSINOPHILIE}

La chute du taux de l'éosinophilie (2\% de $6800 \mathrm{~GB}$ ) constatée le 18 avril 1974 soit 27 jours après le traitement par le thiabendazole semble être plutôt en rapport avec l'élimination partielle ou totale des anguillules, le traitement par la diéthylcarbamazine n'ayant été mise en route que 2 jours auparavant, le 16 avril. La réascension de la courbe à partir du 7 mai et qui s'est maintenue en dents de scie pendant les 10 mois suivants peut faire suggérer que la cure de l'anguillulose n'ait pas été obtenue en dépit de l'absence de larves rhabditoïdes de Strongyloides stercoralis lors des examens coprologiques de contrôle.

Il est difficile d'impliquer la toxocarose dans l'évolution de cette courbe. Tout au plus peut-on constater l'augmentation du taux de l'éosinophilie quelques jours après la prise de Notézine ${ }^{\circledR}$. Celle-ci fut cependant bien discrète et non accompagnée d'hyperleucocytose comme cela est habituellement observée dans les cas de syndromes de Larva migrans viscérale à la suite d'une libération antigénique massive due à la destruction des parasites. On peut évidemment avancer que cette réponse limitée est liée à la 
corticothérapie et aux antihistaminiques associés à la Notézine ${ }^{\circledR}$.

Ce phénomène est en tout cas à rapprocher de la négativité de toutes les réactions séro-immunologiques entreprises aussi bien avant toute corticothérapie qu'après traitement ayant entraîné la disparition des «ascaridés 》 ainsi que de la bonne tolérance générale de ces parasites animaux chez l'homme (incident pulmonaire exclu mais ne pouvant être attribué de façon certaine à la toxocarose).

\section{RÉFÉRENCES}

1. Beaver P. C., Snyder M., Carrera G., Dent J., Lafferty J. : Chronic eosinophilia due to visceral larva migrans. Report of three cases. Pediatrics, 1952, 9, 7-19.

2. Beaver P. C. : The nature of visceral larva migrans. J. Parasitol., 1969, 1, 3-11.

3. Bisseru B., Woodruff A. W., Hutchinson R. J. : Infection with adult Toxocara canis. Brit. Med. J., 1966, 1, 1583-1584.

4. Boettiger C., Werne J. : Ascaris lumbricoides in the human heart. J. Am. Med. Assoc., 1929, 93, 32.

5. Brill R., Churg J., Beaver P. C. : Allergic granulomatosis associated with visceral larva migrans. Am. J. Clin. Pathol., 1953, 23, 1208-1215.

6. Broz O., Trinh P., Cong T. C. : Complications cardiopulmonaires rares et fatales de l'ascaridiose : ascaris dans le cœur et pneumonite par embolisation de leurs œufs. Acta Pathol. Microbiol. Scand., 1960, 23, 36.

7. Brumpt E. : Précis de Parasitologie, vol. 1. Masson, Paris, 1949, 894-896.

8. Draper J. W. : Infection with Lagiochilascaris minor. Br. Med. J., 1963, 6, 931-932.

9. Euzeby J. : Les maladies vermineuses des animaux domestiques et leurs incidences sur la pathologie humaine, tome I, fasc. 1. Vigot, Paris, 1961.

10. Euzeby J. : Les maladies vermineuses des animaux domestiques et leurs incidences sur la pathologie humaine, tome I, fasc. II. Vigot, Paris, 1963.

11. Fox A. S., Kazacos K. R., Gould N. S., Heydemann P. T., Thomas C., Boyer K. M. : Fatal eosinophilie meningoencephalitis and visceral larva migrans caused by the raccoon ascarid. N. Engl. J. Med., 1985, 312, 1619-1623.
12. Gaillard H. : Larva migrans. Gaz. Med. Fr., 1969, 76, 2463-2482.

13. Grinberg A. I. : Rare cases of invasion in man with Toxoscaris leonina and Toxocara mystax. Medicinskaya Parazitol. i. Parazitarn bolesni, Moscou, 1961, 5, 30, 626.

14. Horta J. da S., Delfin J. : Ascaridiose - Migraçao de nunierous vermes adulto pela veia porta; foco de necrose de figado de morfologica particular. Gaz. Med. Port, 1953, 5, 581.

15. Lapierre J., Holler C. : Le syndrome de larva migrans viscérale. Presse Med., 1971, 79, 2163-2166.

16. Leiper R. T. : Two new genera of nematodes occasionnally parasitic in man. Br. Med. J., 1907, 1, 1296-1298.

17. Leon L. A. : Toxocariasis en la infancia. De gacete medica. Guyaquil. Ecuador, 1963, 18, 1-10.

18. Mendheim H., Scheid G., Schmidt J. : Die selteneren Spulwurminfektionen beim Menschen. Z. Tropenmed. Parasitol., 1952, 3, 368-371.

19. Nichols R. L. : The etiology of visceral larva migrans. J. Parasitol., 1956, 42, 349-362.

20. Petithory J. C., Liotet S., Chaumeil C., Moison F., Beddok A., Batellier L., Brumpt L. C. : Le syndrome de larva migrans oculaire. Rev. Fr. Lab., 1990, 207, 69-80.

21. Petithory J. C., Derouin F., Arbogast B., Vandamme M. C., Brumpt L. C. : Données sérologiques sur le syndrome de larva migrans viscéral. Rev. Fr. Labo., 1988, n 178.

22. Petter C. : Étude zoologique de la « Larva migrans ». Ann. Parasitol. Hum. Comp., 1960, 35, 118-137.

23. Petter A. J., Bain O., Orcel L. : Larva migrans expérimentale chez un Primate provoquée par un ascaris de serpent. Ann. Parasitol. Hum. Comp., 1967, 42, 207-210.

24. Phan Trinh: Éclosion larvaire intra-corporelle des œufs d'ascaris erratiques chez un garçon de 4 ans. Pathol. Microbiol., 1965, 28, 443-453.

25. Phouc H. H., Schmauss A. K. : Embolie eines ausgewachsenen Ascaris lumbricoides in du arterua femoralis. Munch. Med. Wshr., 1965, 107, 1482.

26. Rabinovitch I. U. I. A. : A rare case of metastatic ascaridiasis of the cheart and of the large vessels. Sovet. Med., 1957, 21, 117.

27. Sprent J. F. A. : Observation on the development of Toxocara canis in the dog. Parasitology, 1958, 48, 184-209.

28. Wilder M. C. : Nematode endophtalmitis. Trans. Am. Acad., ophtalmo-otolaryngol., 1950, 55, 99-109.

29. Winckel W. E. F., Treurniet A. E. : Infection with Lagiochilascaris minor in man. Docum. Med. géog. et trop. Amsterdam, 1956, 8, 23-28.

30. Woodruff A. W. : Toxocariasis. Br. Med. J., 1970, 3, 663-669. 